\title{
Angular-Resolution and Material-Characterization Measurements for a Dual- Particle Imaging System with Mixed-Oxide Fuel
}

\author{
Alexis Poitrasson-Rivière ${ }^{1}$, J. Kyle Polack ${ }^{1}$, Michael C. Hamel ${ }^{1}$, Dietrich D. Klemm ${ }^{1}$, Kai Ito ${ }^{1}$, \\ Alexander T. McSpaden ${ }^{1}$, Marek Flaska ${ }^{1}$, Shaun D. Clarke ${ }^{1}$, Sara A. Pozzi ${ }^{1}$, Alice Tomanin ${ }^{2}$, and \\ Paolo Peerani ${ }^{3}$ \\ ${ }^{I}$ Department of Nuclear Engineering \& Radiological Sciences, University of Michigan, Ann Arbor, MI 48109 \\ ${ }^{2}$ Lainsa-Italia S.R.L., via E. Fermi 2749, 21027 Ispra (VA), Italy \\ ${ }^{3}$ European Commission, Joint Research Centre, Institute for Transuranium Elements, 21027 Ispra (VA), Italy
}

\begin{abstract}
A dual-particle imaging (DPI) system, capable of simultaneously imaging fast neutrons and gamma rays, has been operated in the presence of mixed-oxide (MOX) fuel to assess the system's angular resolution and materialcharacterization capabilities. The detection principle is based on the scattering physics of neutrons (elastic scattering) and gamma rays (Compton scattering) in organic and inorganic scintillators. The detection system is designed as a combination of a two-plane Compton camera and a neutron-scatter camera. The front plane consists of EJ-309 liquid scintillators and the back plane consists of interleaved EJ-309 and NaI(Tl) scintillators. MCNPX-PoliMi was used to optimize the geometry of the system and the resulting prototype was built and tested using a Cf-252 source as an SNM surrogate. A software package was developed to acquire and process data in real time. The software was used for a measurement campaign to assess the angular resolution of the imaging system with MOX samples. Measurements of two MOX canisters of similar isotopics and intensity were performed for 6 different canister separations (from $5^{\circ}$ to $30^{\circ}$, corresponding to distances of $21 \mathrm{~cm}$ and $131 \mathrm{~cm}$, respectively). The measurements yielded a minimum separation of $20^{\circ}$ at $2.5 \mathrm{~m}$ (86-cm separation) required to see 2 separate hot spots. Additionally, the results displayed good agreement with MCNPX-PoliMi simulations. These results indicate an angular resolution between $15^{\circ}$ and $20^{\circ}$, given the $5^{\circ}$ step. Coupled with its large field of view, and its capability to differentiate between spontaneous fission and $(\alpha, n)$ sources, the DPI system shows its potential for nuclear-nonproliferation applications.
\end{abstract}

Keywords: Compton scattering, neutron elastic scattering, simultaneous detection of gamma rays and neutrons, $\mathrm{NaI}(\mathrm{Tl})$ scintillator, EJ-309 organic-liquid scintillator, MCNPX-PoliMi, mixed-oxide fuel

\section{Introduction}

Reliable techniques to accurately detect, characterize [1], track, and localize [2] special nuclear material (SNM) are needed to support nuclear-nonproliferation efforts. SNM has the particularity of emitting fast neutrons and gamma rays; both signatures should be used to detect SNM efficiently and unambiguously. Some advanced nuclear-nonproliferation efforts include the localization of material of interest, with examples ranging from the localization of a threat object in hostile environments [3] to the localization of nuclear materials in nuclear facilities [4]. The latter is an important part of nuclear-safeguards activities that focus on material accountancy; facilities must be thoroughly scanned to ensure accurate declarations regarding nuclear materials.

To address the aforementioned challenges, a dual-particle imaging (DPI) system capable of simultaneously imaging fast neutrons and gamma rays has been developed [5]. A measurement campaign took place in the spring of 2014 at the Joint Research Centre (JRC) in Ispra, Italy, to assess the angular-resolution and material-characterization capabilities of the DPI system in the presence of nuclear fuel. The DPI system was used to measure two separate mixed-oxide (MOX) 
samples, each with a mass of approximately one kilogram and well-known composition. These samples are category III SNM (SNM of low strategic significance) [6].

\section{Two-Plane DPI System}

\section{A. Concept Design}

The DPI system combines the principles of a two-plane Compton camera and a neutron-scatter camera into a two-plane design with a mixed back plane [7]. EJ-309 and $\mathrm{NaI}(\mathrm{Tl})$ scintillators were selected to induce scatters in the front plane and to obtain gamma-ray absorptions and neutron scatters in the back plane [5]. Pulse shape discrimination is used in the EJ-309 scintillators to classify events as neutron or gamma-ray events [8].

In a two-plane Compton camera, preferable events consist of a scattering in the front plane and an absorption in the back plane $[9,10]$. The measured energy depositions are used to calculate the scattering angle using the Compton formula [11]. The position of an interaction is determined by the position of the detector that recorded the interaction (the center of the detection cell is assumed). The effect of this position uncertainty on the angular resolution will vary with the geometry of the system [12]. These positions are used to create an axis, which, coupled with the scattering angle, is used to create a cone of probable source locations. Reconstructing an image in this way is commonly known as backprojection.

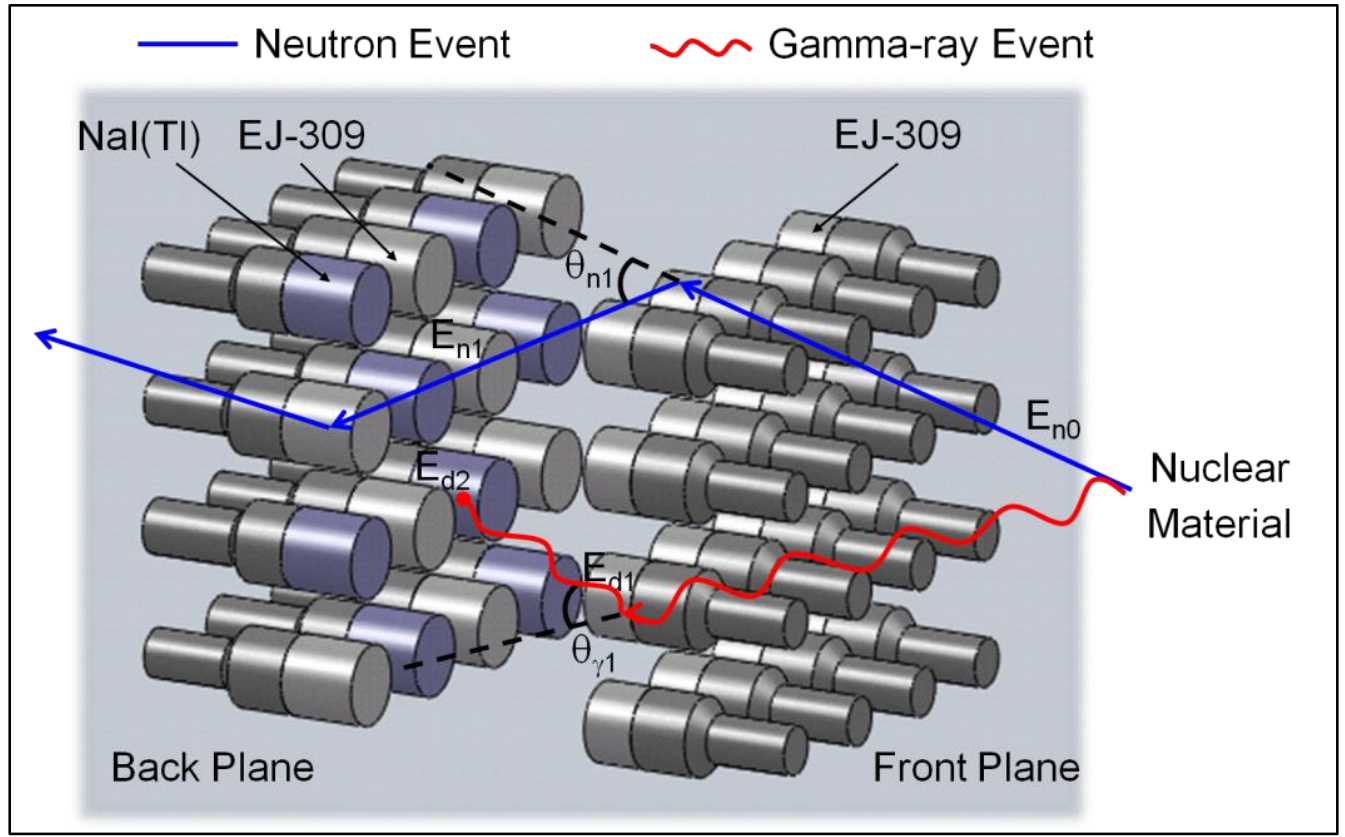

Fig. 1. Diagram of the two-plane DPI system, with gamma-ray and neutron events shown, together with the information used for image reconstruction.

A neutron-scatter camera is based on the elastic-scattering process, and the detection process is similar to that of a Compton camera [13, 14]. Instead of requiring absorptions, however, neutronscatter imaging only requires two scatters and the use of time of flight (TOF) to calculate the energy of the neutron traveling between the planes. Similarly to Compton scattering, the incident neutron energy and the energy deposited are related to the scattering angle. Figure 1 shows an example of neutron and gamma-ray interactions. 
102

103

104 105

The DPI system is based on a particle (neutron or gamma-ray) coincidence measurement. The use of a coincidence system instead of a coded aperture yields a significant advantage for neutronspectroscopy capabilities, while sacrificing overall detection efficiency. Specifically, the measured TOF information enables the user to reconstruct the incoming energy of the neutron. The main drawback is lower detection efficiency, resulting from the necessity for two interactions in two different detection planes.

\section{B. DPI Optimization}

The configuration of detectors in the system shown in Fig. 2 was optimized by using the simulation code MCNPX-PoliMi [15]. The detector sizes were previously selected [5]; the final study was focused on optimizing the distances between the detectors. Three distances were investigated: the separation between the detection planes, the separation between the detectors in the front plane, and the separation between the detectors in the back plane (respectively A, B, and C on Fig. 2). MCNPX-PoliMi simulations were performed for various distances and processed using a dedicated post-processing code [16].

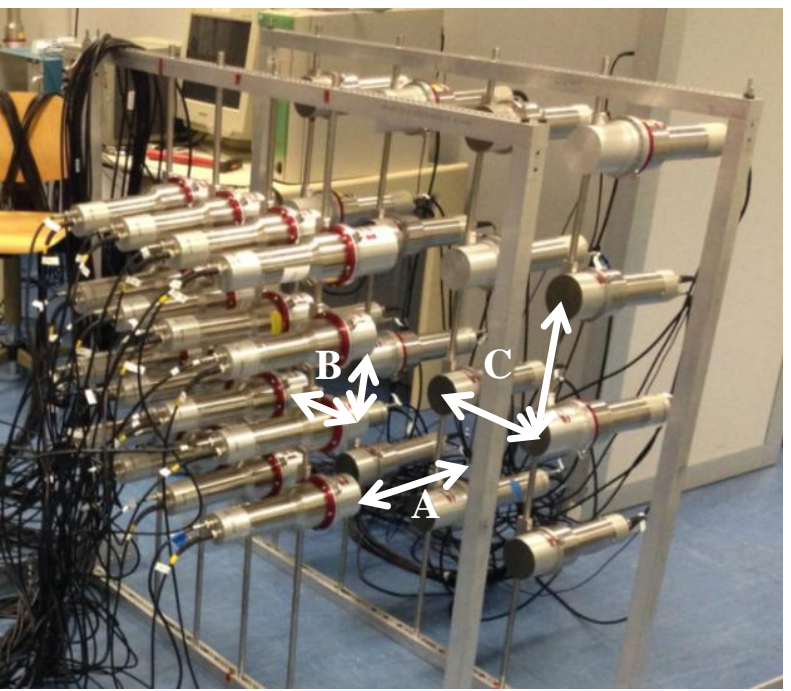

Fig. 2. Optimized DPI system used for the measurement campaign. A, B, and C are the distances investigated in the final optimization study.

Figure 3 shows a flow chart of the post processing applied to the simulation data. MCNPXPoliMi produces list-mode interaction data in the detectors, which is subsequently processed to obtain a realistic simulated detector response. Once detector effects, such as energy and time broadening, have been applied to the interaction data, the simulated detector response can be processed with a correlation algorithm. This algorithm outputs correlated counts that can be imaged through simple backprojection or other imaging techniques. The outlined arrows on Fig. 3 correspond to the inputs (first arrow of each row) and outputs of the post processor. A measured detector response can be provided as input to the post processor, thus providing off-line image reconstruction capabilities for measurements. 


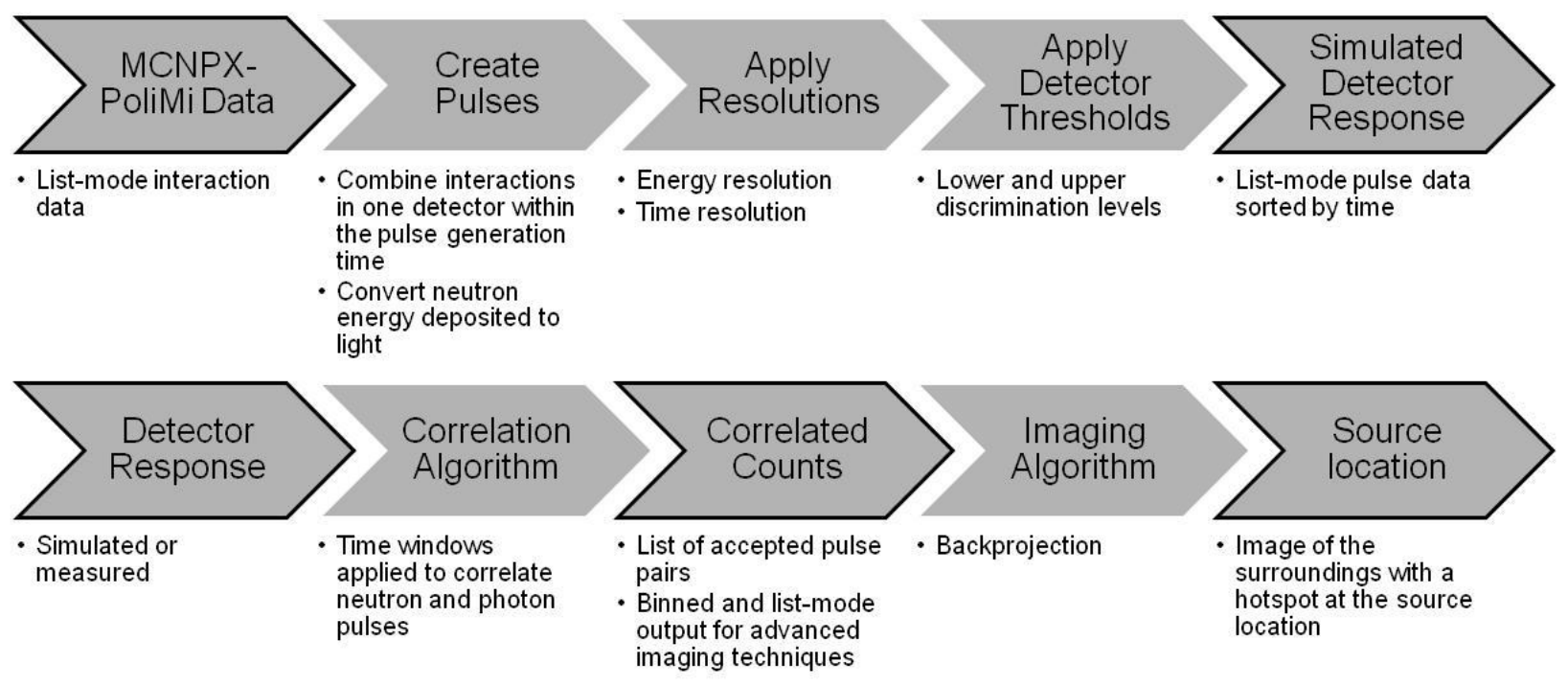

Fig. 3. Flow chart of the MCNPX-PoliMi post processing. The second row of the flow chart can also be used for offline measurement processing.

The optimization study was performed by modeling a single ${ }^{252} \mathrm{Cf}$ point source located $5 \mathrm{~m}$ from the center of the system, on the DPI's axis. $3 \times 10^{9}$ particles (neutron or photon) were emitted in a cone covering the solid angle of the DPI system in each investigated geometrical configuration.

The study focused on two simulated quantities: the number of correlated counts detected by the system - i.e., the DPI's detection efficiency - and the full width half maximum (FWHM) of the image hot spot. The FWHM of the hotspot is calculated without correcting for the baseline contribution of accidental counts to the image. This results in a FWHM larger than the expected resolution. A high detection efficiency and low FWHM is desired. Figures 4 and 5 show the evolution of those parameters for various geometries. The plane separation was varied between 20 and $50 \mathrm{~cm}$, in $10-\mathrm{cm}$ increments. Table 1 shows the intra-plane detector separations investigated in the study. The study investigated separations between 15 and $25 \mathrm{~cm}$. The separations correspond to the distances between two detector centers. Each combination shown in Table 1 was simulated for the four plane separations investigated. Combinations with front-plane separations larger than backplane separations were investigated and yielded inferior results. The evolution of the results of the different intra-plane detector separations followed the same trend for each plane separation. 
Table 1. Distances associated with the geometry index used in Fig. 4.

\begin{tabular}{c|cc}
\hline Geometry Index & $\begin{array}{c}\text { Front-Plane Detector } \\
\text { Separation }(\mathrm{cm})\end{array}$ & $\begin{array}{c}\text { Back-Plane Detector } \\
\text { Separation }(\mathrm{cm})\end{array}$ \\
\hline 1 & 15.0 & 15.0 \\
2 & 15.0 & 17.5 \\
3 & 17.5 & 17.5 \\
4 & 15.0 & 20.0 \\
5 & 17.5 & 20.0 \\
6 & 20.0 & 20.0 \\
7 & 15.0 & 22.5 \\
8 & 17.5 & 22.5 \\
9 & 20.0 & 22.5 \\
10 & 22.5 & 22.5 \\
11 & 15.0 & 25.0 \\
12 & 17.5 & 25.0 \\
13 & 20.0 & 25.0 \\
14 & 22.5 & 25.0 \\
15 & 25.0 & 25.0 \\
\hline
\end{tabular}

Figure 4 shows the intra-plane detector-separation results, which highlight a few geometries that optimize both efficiency and FWHM. The overall trend of the data shows that the system benefits from having a front-plane detector separation smaller than the back-plane one. While only the correlated photon counts are displayed in Fig. $4 \mathrm{~b}$, the correlated neutron counts followed the same trend. For a given back-plane separation, the detection efficiency decreases and angular resolution increases with an increase in front-plane detector separation. The use of a larger separation for the back-plane detectors increases the detection of large-angle scatters. It also decreases the detection probability for small-angle scatters which are likely for energy depositions below threshold in the front plane. For all plane separations, the FWHM trends to a local minimum with a 25 -cm backplane separation and a shorter front-plane separation, while the detection efficiency always trends to a local maximum at the shortest front-plane separation. Therefore, the distances selected were: 15 $\mathrm{cm}$ between the detectors in the front plane and $25 \mathrm{~cm}$ between the detectors in the back plane (geometry index 11). 
(a)

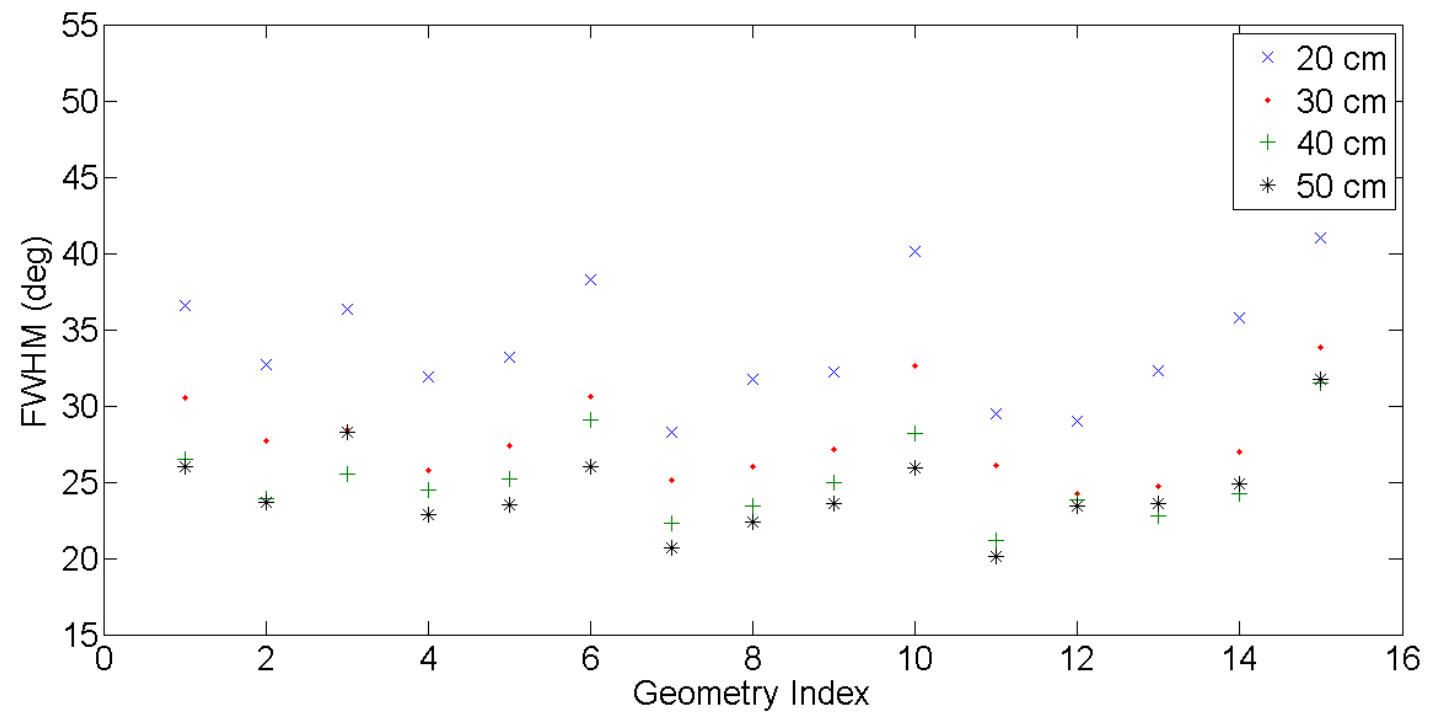

(b)

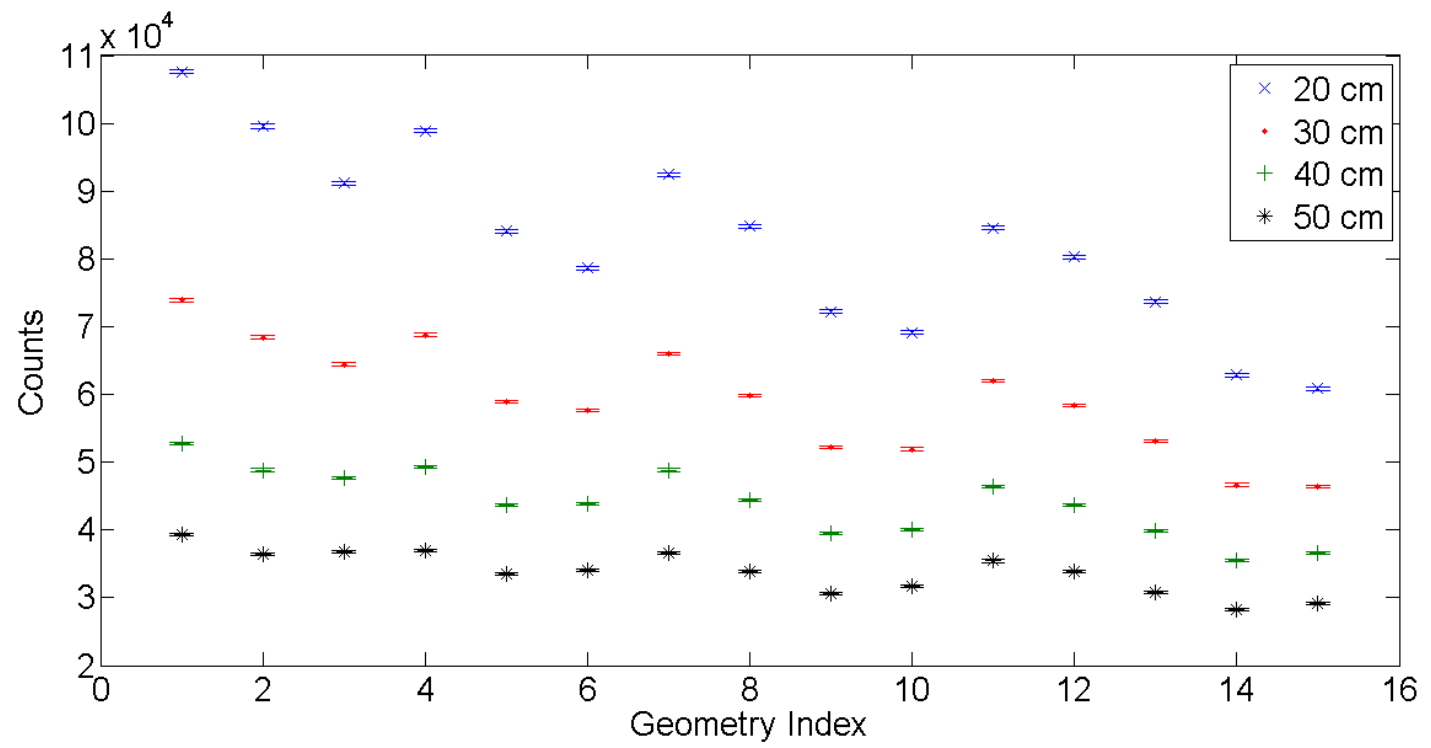

Fig. 4. MCNPX-PoliMi simulation of image hot-spot's FWHM (a) and correlated photon counts (b), for various geometries. The geometry indexes correspond to the intra-plane distances shown in Table I while the distances shown in the legends correspond to the plane separation.

Figure 5 shows the hot-spot FWHM and system efficiency as a function of plane separation. The figure shows that there is a tradeoff between the efficiency and the angular resolution of the system. This result suggest that we can vary the plane separation depending on the requirements: a higher-efficiency application will require the planes close together whereas a higher angular resolution can be achieved by moving the detection planes further apart. A plane separation of 30 $\mathrm{cm}$ was selected as a good tradeoff between the efficiency and the angular resolution of the system. 
(a)
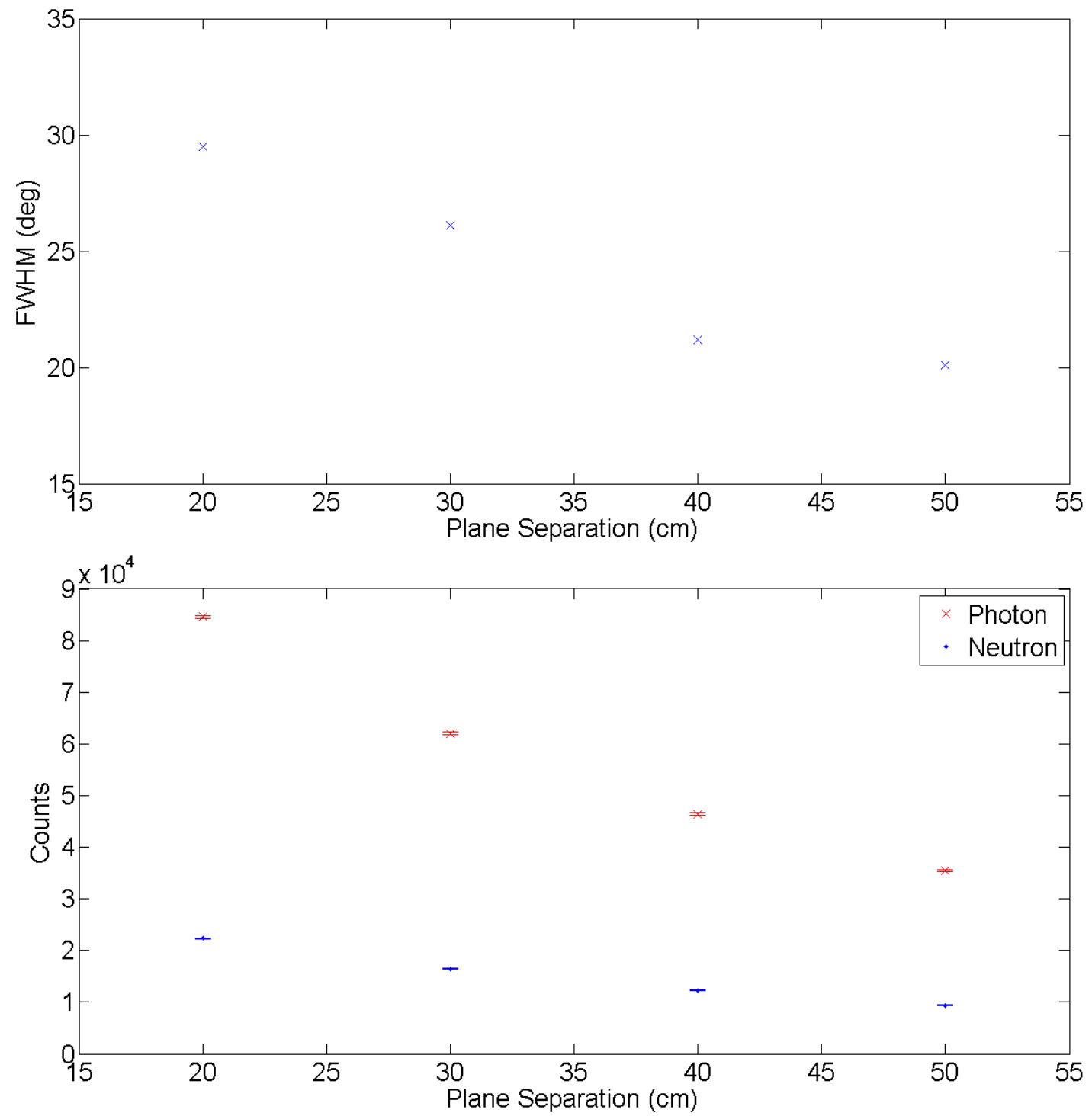

(b)

Fig. 5. MCNPX-PoliMi simulation of image hot-spot FWHM (a) and correlated counts (b), for various plane separations. The intra-plane detector separations used are $15 \mathrm{~cm}$ in the front plane and $25 \mathrm{~cm}$ in the back plane.

Table 2 shows the relative differences of the parameters studied to the selected plane separation. This table highlights how the FWHM varies at a lesser rate than the system efficiency, and seems to be trending to a limit at larger distances.

Table 2. Relative differences of the optimization parameters to the chosen plane separation.

\begin{tabular}{c|ccc}
\hline $\begin{array}{c}\text { Plane } \\
\begin{array}{c}\text { Separation } \\
(\mathrm{cm})\end{array}\end{array}$ & $\begin{array}{c}\text { Relative FWHM } \\
\text { Difference (\%) }\end{array}$ & $\begin{array}{c}\text { Relative Photon } \\
\text { Efficiency Difference (\%) }\end{array}$ & $\begin{array}{c}\text { Relative Neutron } \\
\text { Efficiency Difference (\%) }\end{array}$ \\
\hline 20 & +13.0 & +36.4 & +35.5 \\
30 & 0.0 & 0.0 & 0.0 \\
40 & -18.8 & -25.1 & -25.3 \\
50 & -23.0 & -42.9 & -43.1 \\
\hline
\end{tabular}


Based on the aforementioned optimization studies aimed at finalizing the DPI system's configuration, the detectors in the front plane (5.08-cm thick EJ-309) are separated by $7.48 \mathrm{~cm}$ (15$\mathrm{cm}$ separation minus $7.62-\mathrm{cm}$ detector diameter). The detectors in the back plane $(7.62-\mathrm{cm}$ thick EJ309 and $\mathrm{NaI}(\mathrm{Tl}))$ are separated by $17.48 \mathrm{~cm}(25-\mathrm{cm}$ separation minus $7.62-\mathrm{cm}$ detector diameter). plane detectors had to be replaced by a $7.62-\mathrm{cm}$ thick EJ-309.

\section{Measurement Campaign}

We present the results of experiments performed with the DPI system at the JRC Performance Laboratory (PERLA) in Ispra, Italy in May 2014. The objective of these measurements was to characterize the DPI system in the presence of nuclear fuel. Various SNM samples are available at PERLA, including MOX, Pu oxide, and Pu metal. We focused the testing of the DPI system on 2 MOX canisters of approximately $1 \mathrm{~kg}$ each. Measuring MOX canisters offers two advantages. First, the radiation activity of the MOX sources enabled us to obtain an acceptable amount of data in a limited time (the measurement times were limited to 2 hours). Second, the MOX canisters are also the type of material, in both shape and composition, that the DPI system would be tasked with localizing/characterizing in nuclear-safeguards applications. Both canisters have very similar, wellknown isotopic compositions, shown in Fig. 6a, and neutron production contributions, shown in Fig. 6b, with one canister containing slightly more material than the other. The isotopic abundances of MOX2 are within 2\% of the ones from MOX1. The availability of two sources of similar composition provided us with an opportunity to analyze the angular resolution of the DPI system, as well as to assess its potential for characterizing MOX.

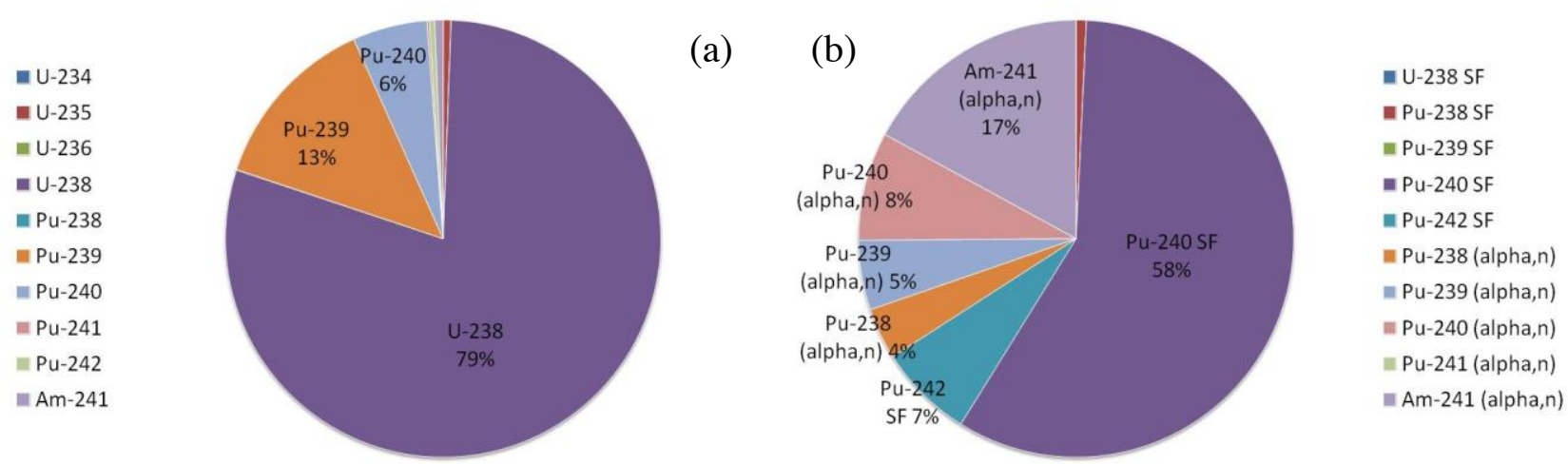

Fig. 6. Isotopic composition of the MOX1 sample (a) and corresponding neutron emission rates (b). SF denotes for spontaneous fission.

\section{A. Angular-Resolution Analysis}

Measurements were set up with the two MOX canisters in front of the system, $2.5 \mathrm{~m}$ away from the center of the system, and angular separations ranging from $5^{\circ}(21.8 \mathrm{~cm})$ to $30^{\circ}(129.4 \mathrm{~cm})$ in $5^{\circ}$ increments. The setups (from $5^{\circ}$ to $30^{\circ}$ ) were respectively measured for $90,105,75,75,120$, and 120 minutes. The variability in these measurement times was due to facility constraints. Fig. 7 is a photograph of the $5^{\circ}$ separation setup as seen from the DPI system. The acquired data were 
processed in real time, which gave us immediate feedback on the DPI system's performance and the opportunity adjust the system if needed.

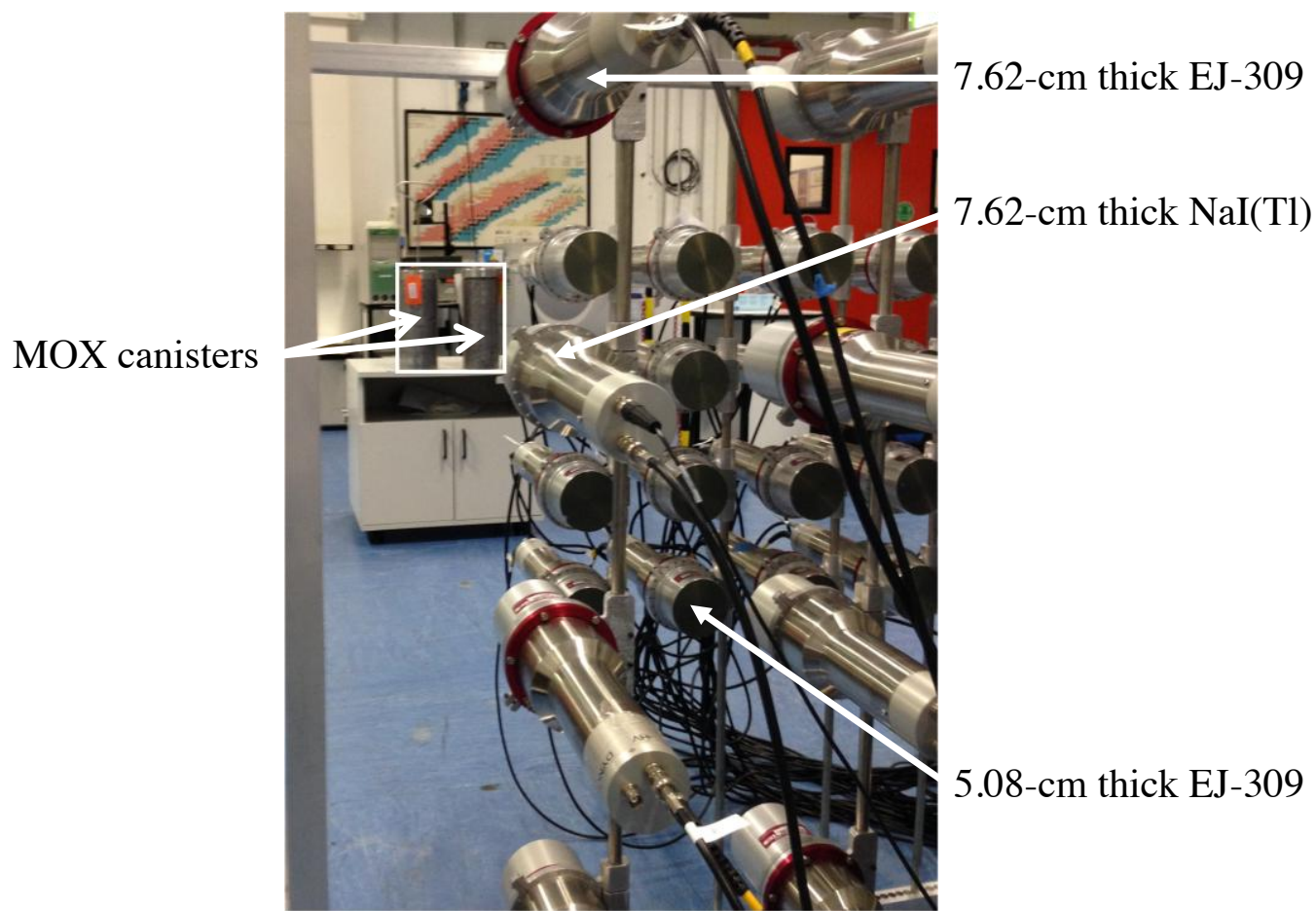

Fig. 7. View of the $2 \mathrm{MOX}$ canisters, with a $5^{\circ}$ separation, placed at a distance of $2.5 \mathrm{~m}$ from the DPI system. The canisters are highlighted with the white square.

Figs. 8 - 10 show the gamma-ray and neutron backprojection images obtained for 5, 15, and $30^{\circ}$ separations, respectively. All the backprojection images were obtained by projecting cones with a $2^{\circ}$ angular thickness on a $2^{\circ}$-meshed sphere. The color bars highlight the number of cones intersecting in a given direction pixel, which we will refer to as the intensity. For each case, both images display hot spots at the source location with the neutron image not fully converged due to the relatively short measurement times. The evolution of the separation is noticeable on the images, as the single hot spot seen for the $5^{\circ}$ separation (Fig. 8) starts extending along the azimuth, going through an oblong shape for the $15^{\circ}$ separation (Fig. 9) and reaching two resolved peaks for the $30^{\circ}$ separation (Fig. 10). 
(a)

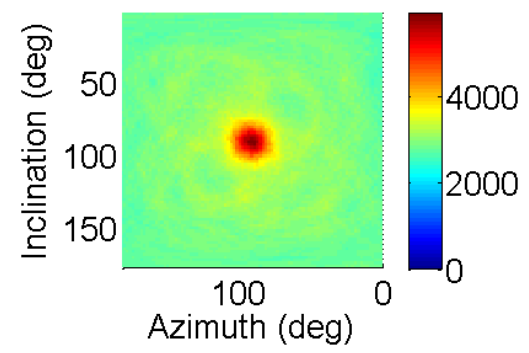

(b)

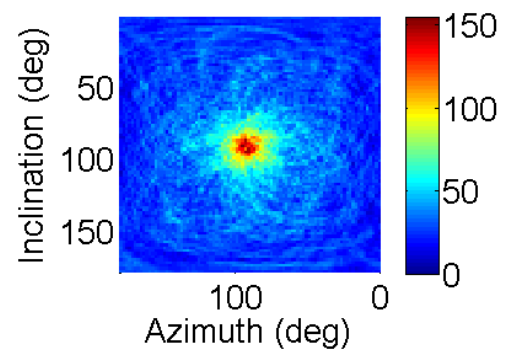

Fig. 8. Gamma-ray (a) and neutron (b) backprojection images of 2 MOX canisters separated by $5^{\circ}$ measured for 90 minutes.

(a)

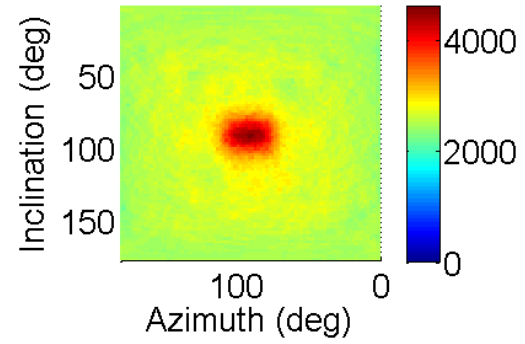

(b)

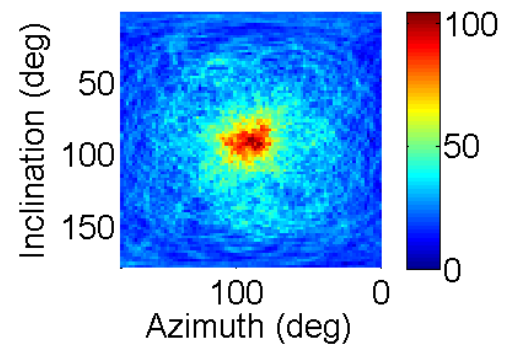

Fig. 9. Gamma-ray (a) and neutron (b) backprojection images of 2 MOX canisters separated by $15^{\circ}$ measured for 75 minutes. 
(a)

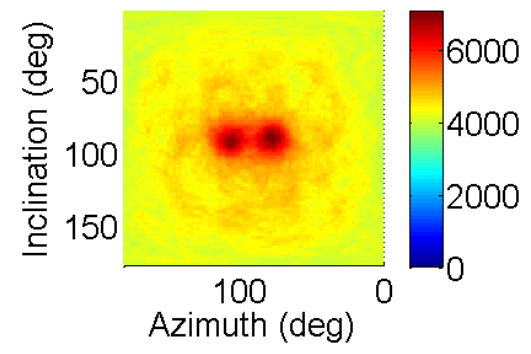

(b)

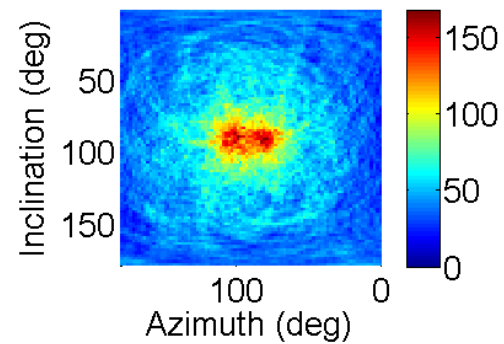

Fig. 10. Gamma-ray (a) and neutron (b) backprojection images of 2 MOX canisters separated by $30^{\circ}$ measured for 120 minutes.

Fig. 11 shows the horizontal slice along the maximum intensity point of the gamma-ray image for each of the six investigated MOX configurations. We can see the formation of the two peaks start at the $20^{\circ}$ separation case with the cases at lesser separation displaying a single hot spot. The $20^{\circ}$ separation case displays two peaks with the left peak reaching $96.5 \%$ of the right peak intensity and a valley between them reaching a low of $93.4 \%$. This result indicates an angular resolution between $15^{\circ}$ and $20^{\circ}$ which does not match the FWHM recorded during the optimization study. This is expected given the FWHM calculation selected.

As the separation between the canisters increases, the valley becomes lower, reaching $84.4 \%$ of the maximum intensity for the $30^{\circ}$ separation, with a left peak at $98.7 \%$ of the maximum intensity. The intensity outside of the defined Gaussian peaks reaches a level of $69 \%$ of the maximum intensity at the base of the peaks. The intensity outside of the Gaussian peaks can be attributed to background as well as accidental correlations such as events that interact in the photomultiplier tubes or do not deposit their full energy. Setting this value as the baseline of the Gaussian peaks, we can notice that the valley reaches the half maximum of the hot spot.

The study could not be carried out for the neutrons due to low statistics. A method to circumvent this is to increase the thickness of the projected cones [17]. This was not further investigated as it would degrade the angular resolution results by increasing the size of the hotspots. Fig. 10b does, however, show two separate hot spots on the neutron image. The neutron image has the advantage of being relatively unaffected by background radiation, allowing sources to be localized using a low number of counts. Advanced imaging methods such as maximum-likelihood expectation-maximization (MLEM) and stochastic origin ensembles are currently being investigated to obtain accurate position information from low-statistic measurements and substantially decrease 250 image noise. 


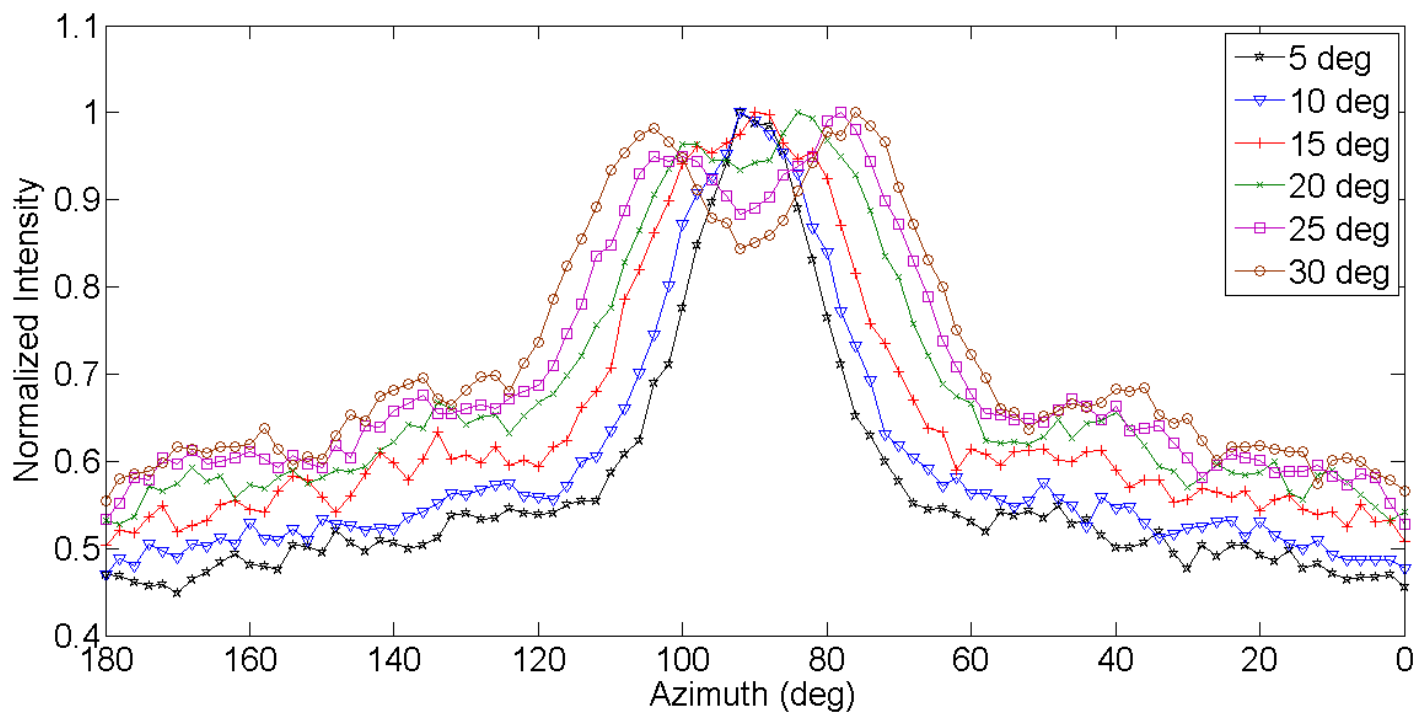

Fig. 11. Gamma-ray backprojection hot-spot slices for all measured angular separations.

\section{B. Simulation Validation}

The relative absence of neutron background also enables us to compare measured to simulated results using our simulation tool. Results show that our simulation tool can accurately simulate the neutrons emitted by MOX. Fig 12 displays the measured and simulated images for a $30^{\circ}$ separation case. The measurement yielded $90 \%$ of the correlated neutron counts recorded in the simulation. We can see matching features on both images, with the intensity of the simulated image slightly higher which matches the discrepancy in the number of correlated neutron counts collected by the system. The clearer separation between the hot spots points to a slight underestimation of the resolution from the simulation. This discrepancy falls within the expected inaccuracies of our model. Those inaccuracies include the uncertainty on the source strength and isotopic concentrations, the uncertainty on the photomultiplier tube model, and the particle misclassification from pulse shape discrimination. 
(a)

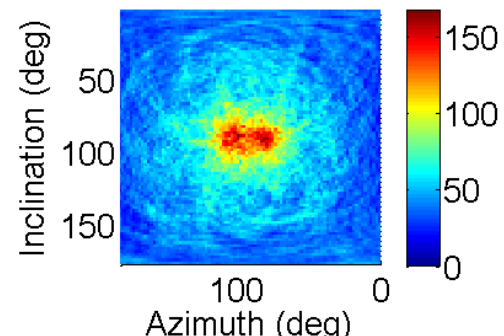

(b)

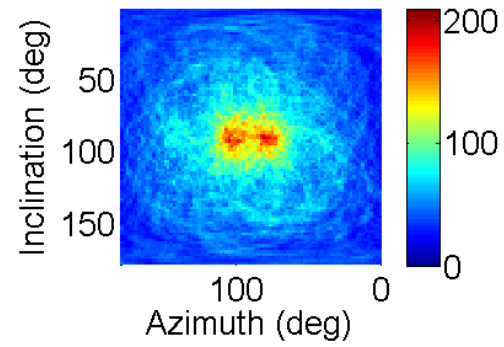

(c)

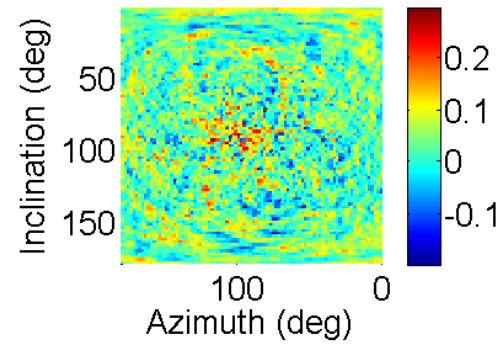

Azimuth (deg)

Fig. 12. Measured (a) and simulated (b) neutron backprojection images for the $30^{\circ}$ separation case and a visualization of the difference between the images normalized to their highest intensity(c).

The measured and simulated neutron energy spectra for the $30^{\circ}$ separation case are shown in Fig. 13. While both spectra are noisy, due to a relatively low number of counts $(\approx 3000$ counts $)$, they both display the same shape. We expect a Watt spectrum for the spontaneous fission neutrons which make up $66 \%$ of the emitted neutrons. The remaining neutrons, which result from $(\alpha, n)$ reactions, are expected to have a higher average energy than the spontaneous fission neutrons. The spectrum collected has a bias towards higher energies due to the relatively high thresholds applied to the detectors. For a single neutron event, both energy depositions must be above the detection threshold to be recorded by the system. While neutron spectra do not have easily recognizable features, the slope of the spectrum between 2.5 and $6 \mathrm{MeV}$ can be used to discern a pure spontaneous fission source from a mixed source like the MOX canisters [18]. This characterization can also be done by looking at single-detector neutron pulse height distributions (PHD) [19]. 


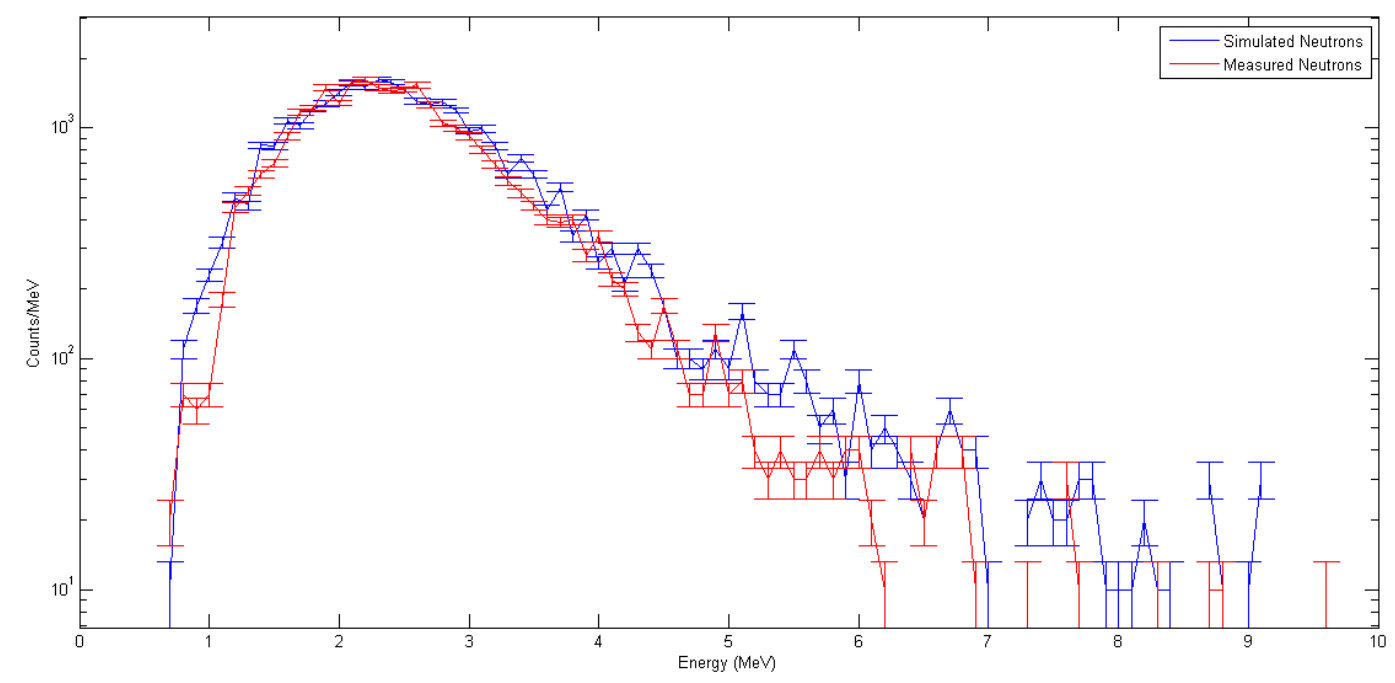

Fig. 13. TOF-derived neutron spectrum from the $30^{\circ}$ separation case. The spectrum is obtained from the energy deposited in the first scatter and the subsequent time of flight.

(a)

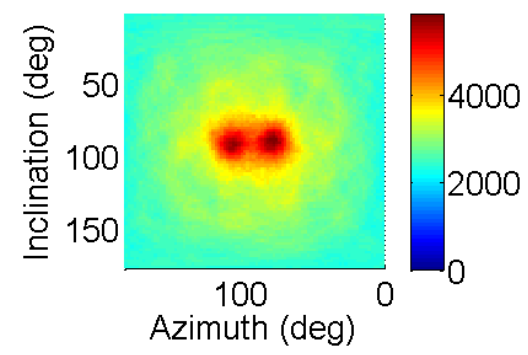

(b)

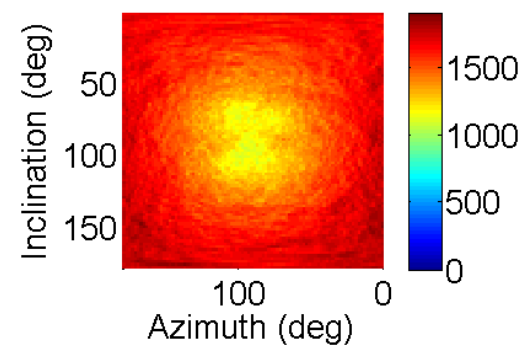

Fig. 14. Gamma-ray backprojection images for the summed energies between 300 and $800 \mathrm{keV}$ (a) and for any other summed energies (b). 


\section{Summary and Conclusions}

A two-plane DPI system able to simultaneously image fast neutrons and gamma rays was developed. It consists of a front plane made of organic-liquid scintillators to detect gamma-ray and neutron scatters, and a back plane made of EJ-309 with $\mathrm{NaI}(\mathrm{Tl})$ to induce neutron scatters and gamma-ray photoelectric absorptions. The DPI system's geometry was optimized using MCNPXPoliMi to maximize detection efficiency while optimizing the angular resolution. The DPI system was subsequently characterized by measuring MOX samples at the JRC PERLA facility in Ispra, Italy.

Measurements of two well-characterized MOX canisters placed at a distance of $2.5 \mathrm{~m}$ and with separation ranging from $5^{\circ}$ to $30^{\circ}$ were performed with the DPI system. The minimum separation that yielded two separate hot spots on the gamma-ray backprojection image was found to be approximately $20^{\circ}$. Given our $5^{\circ}$ step, we can conclude that the angular resolution of the DPI system is between $15^{\circ}$ and $20^{\circ}$, which. While the $30^{\circ}$ case yielded a neutron image with two separate hotspots, the neutron images do not have enough counts to accurately obtain an angular resolution measurement. The comparison of measured and simulated results validated the use of our simulation tool for the optimization of the DPI system.

The system has demonstrated the ability to distinguish MOX canisters with an angular separation of at least $20^{\circ}$, which, for a distance to the source of $2.5 \mathrm{~m}$, equates $86 \mathrm{~cm}$. The system should be appropriate for the preliminary localization of hot zones in nuclear facilities. The approach presented in this paper has also shown source characterization capabilities using both neutron and gamma-ray information. The neutron information, using the TOF-derived neutron spectrum or single-detector PHDs, has the advantage of being relatively unaffected by background. The photon spectral information needs background subtraction, which was performed in this work by coupling it with the position information. Both of these tasks are of interest for nuclearsafeguards applications. Advanced imaging and data-analysis methods are currently being investigated to further enhance the DPI system.

\section{Acknowledgment}

This work is supported, in-part, by the National Nuclear Security Administration through NA22 funding opportunity DE-FOA-0000568. It is also supported in part by the National Science Foundation and the Domestic Nuclear Detection Office of the Department of Homeland Security through the Academic Research Initiative Award \# CMMI 0938909 as well as by the Sandia National Laboratories Excellence in Engineering Research Fellowship. The prototype system is funded by the Department of Energy, Nuclear Energy University Program, Award \# DENE0000324.

\section{References}

[1] J. L. Dolan, M. Flaska, A. Poitrasson-Riviere, A. Enqvist, P. Peerani, D. L. Chichester, Sara A. Pozzi "Plutonium measurements with a fastneutron multiplicity counter for nuclear safeguards applications," Nucl. Instr. Meth. A, vol. 763, pp. 565 - 574, 2014.

[2] K. P. Ziock, W. W. Craig, L. Fabris, R. C. Lanza, S. Gallagher, B. K. P. Horn, N. W. Madden, "Large area imaging detector for long-range, passive detection of fissile material," IEEE Trans. Nucl. Sci., vol. 51, pp. 2238 - 2244, 2004.

[3] S. Zelakiewicz, R. Hoctor, A. Ivan, W. Ross, E. Nieters, W. Smith, D. McDevitt, M. Wittbrodt, B. Millbrath, "SORIS-A standoff radiation imaging system," Nucl. Instr. Meth. A, vol. 652, pp. 5-9, 2011.

[4] Y. Morishita, S. Yamamoto, K. Izakib, J. H. Kaneko, K. Toic, Y. Tsubota, "Development of a Si-PM based alpha camera for plutonium detection in nuclear fuel facilities," Nucl. Instr. Meth. A, vol. 747, pp. $81-86,2014$.

[5] A. Poitrasson-Rivière, M. C. Hamel, J. K. Polack, M. Flaska, S. D. Clarke, S. A. Pozzi, "Dual-Particle Imaging System based on Simultaneous Detection of Photon and Neutron Collision Events," Nucl. Instr. Meth. A, vol. 760, pp. 40 - 45, 2014 
[6] http://www.nrc.gov/security/domestic/mca/snm.html

[7] M. C. Hamel, A. Poitrasson-Rivière, J. K. Polack, S. D. Clarke, M. Flaska, S. A. Pozzi, "Design and Analysis of a Two-Plane Dual-Particle Imaging System," Proceedings of the Institute of Nuclear Materials Management 54th Annual Meeting on CD-ROM, Palm Desert, California, USA. July $14-18,2013$.

[8] J. K. Polack, M. Flaska, A. Enqvist, C. S. Sosa, C. C. Lawrence, S. A. Pozzi, "An Algorithm for Charge-Integration, Pulse-Shape Discrimination and Estimation of Neutron/Photon Misclassification in Organic Scintillators," accepted for publication in Nucl. Instr. Meth. A

[9] R. W. Todd, J. M. Nightingale, D. B. Everett, “A proposed gamma camera,” Nature, vol. 251, no. 5471, pp. 132 - 134, 1974.

[10] M. Singh, "An Electronically Collimated Gamma Camera for Single Photon Emission Computed Tomography. Part I: Theoretical Considerations and Design Criteria," Med. Phys., vol. 10, pp. $421-427,1983$.

[11] G. F. Knoll, "Radiation Detection and Measurement (4 ${ }^{\text {th }}$ Ed.)." John Wiley \& Sons, 2010.

[12] C. E. Ordonez, W. Chang, and A. Bolozdynya, "Angular Uncertainties due to Geometry and Spatial Resolution in Compton Cameras," IEEE Trans. Nucl. Sci., vol. 46, pp. 1142-1147, 1999.

[13] N. Mascarenhas, J. Brennan, K. Krenz, P. Marleau, S. Mrowka, "Results with the neutron scatter camera," IEEE Trans. Nucl. Sci., vol. 56, pp. $1269-1273,2009$.

[14] R. S. Miller, J. R. Macri, M. L. McConnell, J. M. Ryan, E. Flückiger, L. Desorgher, "SONTRAC: An imaging spectrometer for MeV neutrons," Nucl. Instr. Meth. A, vol. 505, pp. $36-40,2003$.

[15] S. A. Pozzi, S. D. Clarke, W. Walsh, E. C. Miller, J. Dolan, M. Flaska, B. M. Wieger, A. Enqvist, E. Padovani, J. K. Mattingly, D. Chichester, P. Peerani, "MCNPX-PoliMi for Nuclear Nonproliferation Applications," Nucl. Instr. Meth. A, vol. 694, pp. 119-125, 2012

[16] E. C. Miller, S. D. Clarke, M. Flaska, S. A. Pozzi, "MCNPX-PoliMi Post-processing Algorithm for Detector Response Simulations," Journal of Nuclear Materials Management, Winter 2012, XL, 2, pp. $34-41,2012$

[17] M. J. Myjak, C. E. Seifert, "Real-Time Compton Imaging for the GammaTracker Handheld CdZnTe Detector." IEEE Trans. Nucl. Sci., vol. 55, pp. $769-777,2008$.

[18] M. C. Hamel, J. K. Polack, A. Poitrasson-Rivière, D. D. Klemm, M. Flaska, S. D. Clarke, S. A. Pozzi, A. Tomanin, P. Peerani, “Time-of-Flight Neutron Spectrum Unfolding for Mixed-Oxide Nuclear Fuel and Plutonium Metal Using a Dual-Particle Imager," IEEE Nuclear Science Symposium and Medical Imaging Conference Record, November 8-15, 2014, Seattle, Washington, USA.

[19] J. L. Dolan, M. Flaska, Sara A. Pozzi, D. L. Chichester, "Passive measurements of mixed-oxide fuel for nuclear nonproliferation," Nucl. Instr. Meth. A, vol. 703,pp. $102-108,2013$.

[20] D. Xu, Z. He, “Gamma-ray energy-imaging integrated spectral deconvolution,” Nucl. Instr. Meth. A, vol. 574 , pp. 98 - 109 , 2007. 


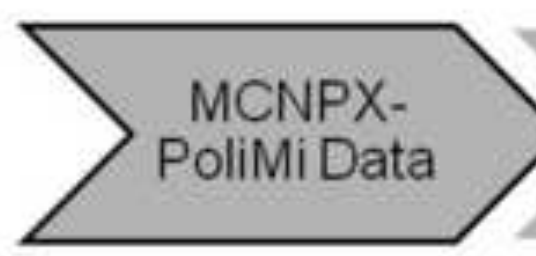

- List-mode interaction data

- Combine interactions in one detector within the pulse generation time

- Convert neutron energy deposited to light

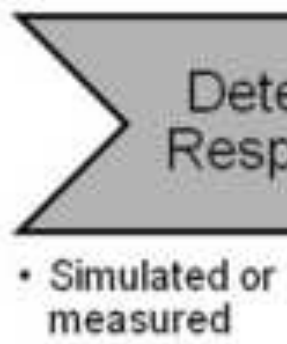
measured

\section{Create Pulses}

Energy resolution

- Time resolution

- Lower and upper discrimination levels

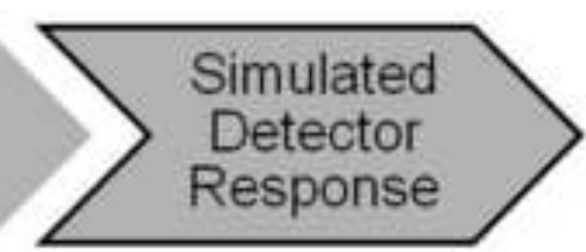

- List-mode pulse data sorted by time

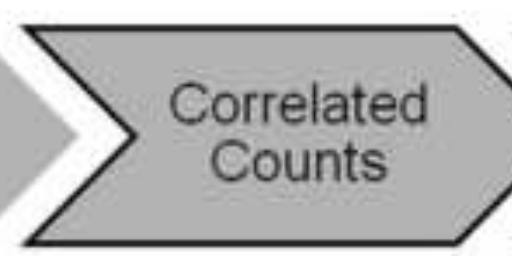

- List of accepted pulse pairs

- Binned and list-mode output for advanced imaging techniques
- Time windows applied to correlate neutron and photon pulses

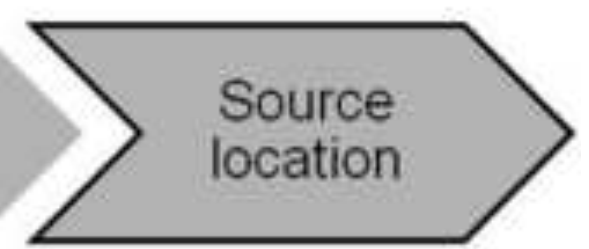

- Image of the surroundings with a hotspot at the source location 